九州大学学術情報リポジトリ

Kyushu University Institutional Repository

\title{
MULTISTAGE MARKOV DECISION PROCESSES WITH MINIMUM CRITERIA OF RANDOM REWARDS
}

Otsubo, Yoshio

Department of Mathematics and Information Science, Faculty of Science, Kochi University

https://doi.org/10.5109/12598

出版情報: Bulletin of informatics and cybernetics. 38, pp.15-25，2006-12. Research Association of Statistical Sciences

バージョン：

権利関係 : 
MULTISTAGE MARKOV DECISION PROCESSES WITH MINIMUM CRITERIA OF RANDOM REWARDS

by

Yoshio Онтsuво

Reprinted from the Bulletin of Informatics and Cybernetics Research Association of Statistical Sciences, Vol.38

FUKUOKA, JAPAN

2006 


\title{
MULTISTAGE MARKOV DECISION PROCESSES WITH MINIMUM CRITERIA OF RANDOM REWARDS
}

\author{
By
}

\section{Yoshio Онтsuво*}

\begin{abstract}
We consider multistage decision processes where criterion function is an expectation of minimum function. We formulate them as Markov decision processes with imbedded parameters. The policy depends upon a history including past imbedded parameters, and the rewards at each stage are random and depend upon current state, action and a next state. We then give an optimality equation by using operators and show that there exists a right continuous deterministic Markov policy, which depends upon a current state and an imbedded parameter.
\end{abstract}

Key Words and Phrases: Existence of optimal policy, Invariant imbedding method, Markov decision process, Minimum criteria, Optimality equation.

\section{Introduction}

We consider a generalization of stochastic decision-making in a fuzzy environment. Bellman and Zadeh (1970) first introduce multistage decision processes in a fuzzy environment and propose a recursive formula for deterministic processes and stochastic processes. However Iwamoto and Fujita (1995) and Iwamoto et al. (1999) point out that Bellman and Zadeh's stochastic recursive formula is a posteriori conditional decision process, whose criterion is

$$
r_{1}\left(a_{1}\right) \wedge E^{a_{1}}\left[r_{2}\left(a_{2}\right) \wedge E^{a_{2}}\left[r_{3}\left(a_{3}\right) \wedge \cdots \wedge E^{a_{N-2}}\left[r_{N-1}\left(a_{N-1}\right) \wedge E^{a_{N-1}}\left[r_{N}\left(x_{N}\right)\right]\right] \cdots\right]\right],
$$

where $a_{1}, a_{2}, \ldots, a_{N-1}$ are actions (controls), $r_{1}, r_{2}, \ldots, r_{N-1}$ are reward (membership) functions on an action space, $r_{N}$ is a reward (goal) function on a state space, $x_{N}$ is a state, $E^{a_{i}}$ is a conditional expectation operator given an action $a_{i}$ and $\wedge$ denotes the minimum operator. Iwamoto and Fujita (1995) investigate a regular decision process with a criterion

$$
E_{x_{1}}^{\pi}\left[r_{1}\left(a_{1}\right) \wedge r_{2}\left(a_{2}\right) \wedge \cdots \wedge r_{N-1}\left(a_{N-1}\right) \wedge r_{N}\left(x_{N}\right)\right]
$$

where $x_{1}$ is an initial state and $\pi$ is a policy, and Iwamoto et al. (1999) consider a generalized decision process with a criterion

$$
E_{x_{1}}^{\pi}\left[r_{1}\left(x_{1}, a_{1}\right) \circ r_{2}\left(x_{2}, a_{2}\right) \circ \cdots \circ r_{N-1}\left(x_{N-1}, a_{N-1}\right) \circ r_{N}\left(x_{N}\right)\right],
$$

\footnotetext{
* Department of Mathematics and Information Science, Faculty of Science, Kochi University, 2-5-1 Akebono-cho, Kochi 780-8520, Japan. E-mail: ohtsubo@math.kochi-u.ac.jp
} 
$r_{1}, r_{2}, \ldots, r_{N-1}$ are membership functions of a state and a action, and $\circ$ is an associative binary relation which includes the minimum relation $\wedge$ as a special case. Both give an optimality equation as a recursive formula and find an optimal Markov policy by using an invariant imbedding method. When $\circ=\wedge$, Iwamoto et al. (2001) show that an optimal Markov policy for an invariant imbedding approach yields an optimal general policy which depends upon the history consisting of only states and actions, and give an example in which there is no optimal Markov policy in a space of policies for general decision problem, in which an invariant imbedding approach is not used.

In such a fuzzy environment Kacprzyk (1978) and Esogbue and Bellman (1984) investigate multistage decision processes with fuzzy termination time. Also White (1993a), Wu and Lin (1999) and Ohtsubo and Toyonaga (2002) consider a minimizing problem of threshold probability in discounted Markov decision processes by using a kind of invariant imbedding method, and Ohtsubo (2003) applies them to stochastic shortest path problem.

In this paper we consider multistage decision processes with a countable state space and a minimum criterion

$$
E_{x_{1}}^{\pi}\left[r_{1}\left(x_{1}, a_{1}, x_{2}\right) \wedge r_{2}\left(x_{2}, a_{2}, x_{3}\right) \wedge \cdots \wedge r_{N-1}\left(x_{N-1}, a_{N-1}, x_{N}\right) \wedge r_{N}\left(x_{N}, a_{N}\right)\right],
$$

where $r_{1}, r_{2}, \ldots, r_{N-1}$ are random variables depending upon not only a current state and a current action but also a next state. By the way, $r_{i}\left(a_{i}\right)$ in Bellman and Zadeh (1970) and Iwamoto and Fujita (1995) and $r_{i}\left(x_{i}, a_{i}\right)$ in Iwamoto et al. (1999) are both deterministic but not random. However $r_{i}\left(x_{i}, a_{i}, x_{i+1}\right)$ in our problem is random. Also, in all references as above, policies depend upon only current state and action, but our policies depend upon a past history. We then show that an optimal value function for imbedded processes satisfies an optimality equation (recursive formula) by using operators and give an optimal (imbedded) Markov policy which is right continuous with respect to imbedded parameter.

\section{Notations and formulation}

In this section we formulate our optimization problems as multistage Markov decision Processes $\Gamma=\left(\left(X_{n}\right),\left(A_{n}\right),\left(Y_{n}\right), p_{n}\right)$ with a finite discrete time space $\mathbf{N}=$ $\{1,2, \ldots N\}$. The state space $S$ is countable, and denote the state at time $n \in \mathbf{N}$ by $X_{n}$. The action space $A=\cup_{s \in S} A(s)$ is countable, where $A(s)$ is a nonempty set of admissible finite actions when the system is in state $s \in S$, and denote the action at time $n \in \mathbf{N}$ by $A_{n}$. The reward space $E$ is a countable set $\left\{y_{1}, y_{2}, \ldots\right\}$ where each reward $y_{i}(i=1,2, \ldots)$ is nonnegative and $E$ is bounded, that is, $0 \leq y_{i} \leq M$ for some $M>0$ and every $y_{i} \in E$. If $M \leq 1$, we are in a fuzzy environment. $Y_{n} \in E$ is a random reward function at time $n \in \mathbf{N}$ and we define time-nonstationary probability distributions by

$$
\begin{aligned}
& q_{n}^{a}\left(s^{\prime} \mid s\right)=P\left(X_{n+1}=s^{\prime} \mid X_{n}=s, A_{n}=a\right), \quad 1 \leq n \leq N-1, \\
& \hat{q}_{n}^{a}\left(y \mid s, s^{\prime}\right)=P\left(Y_{n}=y \mid X_{n}=s, X_{n+1}=s^{\prime}, A_{n}=a\right), \quad 1 \leq n \leq N-1, \\
& \tilde{p}_{N}^{a}(y \mid s)=P\left(Y_{N}=y \mid X_{N}=s, A_{N}=a\right)
\end{aligned}
$$

and set

$$
p_{n}^{a}\left(s^{\prime}, y \mid s\right)=q_{n}^{a}\left(s^{\prime} \mid s\right) \hat{q}_{n}^{a}\left(y \mid s, s^{\prime}\right)=P\left(X_{n+1}=s^{\prime}, Y_{n}=y \mid X_{n}=s, A_{n}=a\right)
$$


for $s, s^{\prime} \in S, a \in A(s)$ and $y \in E$, where $\sum_{s^{\prime} \in S} q_{n}^{a}\left(s^{\prime} \mid s\right)=\sum_{y \in E} \hat{q}_{n}^{a}\left(y \mid s, s^{\prime}\right)=\sum_{y \in E} \tilde{p}_{N}^{a}(y \mid s)$ $=1$. For an additive case, it is known in Chapter 7 of Howard (1960) and Chapter 1 of White (1993b) that the right-side of an optimality equation is

$$
\sum_{s^{\prime} \in S} \sum_{y \in E} p_{n}^{a}\left(s^{\prime}, y \mid s\right)\left(y+v\left(s^{\prime}\right)\right)=\sum_{s^{\prime} \in S} \sum_{y \in E} p_{n}^{a}\left(s^{\prime}, y \mid s\right) y+\sum_{s^{\prime} \in S} q_{n}^{a}\left(s^{\prime} \mid s\right) v\left(s^{\prime}\right)
$$

for some function $v$, which implies that one-step expected reward $\hat{r}_{s}^{a}=$ $\sum_{s^{\prime} \in S} \sum_{y \in E} p_{n}^{a}\left(s^{\prime}, y \mid s\right) y$ depend upon a state $s$ and an action $a$. Hence in an additive case it is enough to give one-step reward $\hat{r}_{s}^{a}$, which is not random. However in our minimum case we generally have

$$
\sum_{s^{\prime} \in S} \sum_{y \in E} p_{n}^{a}\left(s^{\prime}, y \mid s\right)\left(y \wedge v\left(s^{\prime}\right)\right) \neq \sum_{s^{\prime} \in S} \sum_{y \in E} p_{n}^{a}\left(s^{\prime}, y \mid s\right) y \wedge \sum_{s^{\prime} \in S} q_{n}^{a}\left(s^{\prime} \mid s\right) v\left(s^{\prime}\right) .
$$

Thus our decision problem is a generalization of Bellman and Zadeh (1970), Iwamoto and Fujita (1995) and Iwamoto et al. (2001).

We define the random reward as a criterion function by

$$
Z=\min _{1 \leq n \leq N} Y_{n}
$$

Then our problem is to maximize the expected reward $E_{s}^{\pi}[Z]$ with respect to all policies $\pi$. In order to analysis our problem we define the random reward for a subproblem by

$$
Z_{n}=\min _{n \leq k \leq N} Y_{k}, \quad 1 \leq n \leq N
$$

Further we define another random sequence as an imbedded parameter by

$$
\Lambda_{1}=\lambda, \quad \Lambda_{n+1}=\min \left(Y_{n}, \Lambda_{n}\right), \quad 1 \leq n \leq N,
$$

where $\lambda$ is a given initial value in $[0, M]$.

We use $S_{1}=S \times[0, M]$ as a new state space. Let $H_{1}=S_{1}$ and $H_{n+1}=H_{n} \times$ $A \times S_{1}$ for each $1 \leq n \leq N-1$. Then $H_{n}$ represents the set of all possible histories $h_{n}=\left(s_{1}, \lambda_{1}, a_{1}, s_{2}, \lambda_{2}, \ldots, a_{n-1}, s_{n}, \lambda_{n}\right)$ of the system when the $n$th action must be chosen, and we denote by $\theta_{n}$ the history at time $n \in \mathbf{N}$. A decision rule $\delta_{n}$ for time $n \in \mathbf{N}$ is a conditional probability given $\theta_{n}=\left(X_{1}, \Lambda_{1}, A_{1}, X_{2}, \Lambda_{2}, \ldots, A_{n-1}, X_{n}, \Lambda_{n}\right)$ : $\delta_{n}\left(a_{n} \mid h_{n}\right)=P\left(A_{n}=a_{n} \mid \theta_{n}=h_{n}\right)$. It is assumed that $\delta_{n}\left(A_{n} \in A\left(s_{n}\right) \mid h_{n}\right)=1$ for every history $h_{n}=\left(s_{1}, \lambda_{1}, a_{1}, s_{2}, \lambda_{2}, \ldots, s_{n}, \lambda_{n}\right) \in H_{n}$ and $\delta_{n}\left(a_{n} \mid \cdot\right)$ is Lebesgue-Stieltjes measurable function on $H_{n}$. We denote by $\Delta(n)$ the set of such decision rules $\delta_{n}$. A policy $\pi$ is a finite sequence of decision rules $\left(\delta_{n}, n \in \mathbf{N}\right)=\left(\delta_{1}, \delta_{2}, \ldots, \delta_{N}\right)$, where $\delta_{n} \in \Delta(n)$. We denote by $C$ the set of all such policies. We also denote by $C_{n}^{N}$ the set of all sequences $\left(\delta_{n}, \delta_{n+1}, \ldots, \delta_{N-1}\right)$ of decision rules $\delta_{k} \in \Delta(k-n+1)(k=n, n+1, \ldots, N)$. $C_{n}^{N}$ are used in subproblems. We then note that $C_{1}^{N}=C$.

A policy $\pi=\left(\delta_{n}, n \in \mathbf{N}\right)$ is said to be Markov (for an imbedded decision process) when the decision rule $\delta_{n}$ is a function of $\left(X_{n}, \Lambda_{n}\right)=\left(s_{n}, \lambda_{n}\right)$ for every $n \in \mathbf{N}$. We denote the set of such decision rules by $\Delta_{M}$ and the set of all Markov policies by $C_{M}$. Also, a policy $\pi=\left(\delta_{n}, n \in \mathbf{N}\right)$ is called a deterministic Markov policy if $\pi$ is Markov and $\delta_{n}(a \mid s, \lambda)=1$ for every $(s, \lambda) \in S_{1}$ and some $a \in A(s)$. We write $\delta_{n}(s, \lambda)=a$ for such a decision rule $\delta_{n}$ and we denote by $\Delta_{D}$ the set of such decision rules. We also 
denote the set of all deterministic Markov policies by $C_{D} . \pi \in C_{n}^{N} \cap C_{D}$ means that $\pi=\left(\delta_{n}, \delta_{n+1}, \ldots, \delta_{N}\right) \in C_{n}^{N}$ and $\delta_{k} \in \Delta_{D}(k=n, n+1, \ldots, N)$.

A decision rule $\delta \in \Delta_{D}$ is said to be right continuous (on $S_{1}$ ) if for each $(s, \lambda) \in S_{1}$ there is a positive real number $\mu$ such that $\delta(s, \lambda)=\delta(s, \lambda+u)$ for all $u: 0 \leq u<\mu$. A policy $\pi=\left(\delta_{n}, n \in \mathbf{N}\right) \in C_{D}$ is said to be right continuous if the decision rule $\delta_{n}$ is right continuous for every $n \in \mathbf{N}$.

We consider an imbedded decision subproblem in which we maximize an expectation

$$
F_{n}^{\pi}(s, \lambda)=E_{s}^{\pi}\left[\lambda \wedge Z_{n}\right], \quad n \in \mathbf{N}
$$

with respect to all policies $\pi$ for any imbedded parameter $\lambda \in[0, M]$ where $x \wedge y=$ $\min (x, y)$. When $N=3$, the explicit form of the expectation $F_{1}^{\pi}\left(s_{1}, \lambda\right)$ is

$$
\begin{aligned}
E_{s_{1}}^{\pi}\left[\lambda \wedge Z_{1}\right]= & \sum_{a_{1} \in A\left(s_{1}\right)} \sum_{y_{1} \in E} \sum_{s_{2} \in S} \sum_{a_{2} \in A\left(s_{2}\right)} \sum_{y_{2} \in E} \sum_{s_{3} \in S} \sum_{a_{3} \in A\left(s_{3}\right)} \sum_{y_{3} \in E}\left(\lambda \wedge y_{1} \wedge y_{2} \wedge y_{3}\right) \\
& \times \tilde{p}_{3}^{a_{3}}\left(y_{3} \mid s_{3}\right) \delta_{3}\left(a_{3} \mid s_{1}, \lambda, a_{1}, s_{2}, \lambda \wedge y_{1}, a_{2}, s_{3}, \lambda \wedge y_{1} \wedge y_{2}\right) \\
& \times p_{2}^{a_{2}}\left(s_{3}, y_{2} \mid s_{2}\right) \delta_{2}\left(a_{2} \mid s_{1}, \lambda, a_{1}, s_{2}, \lambda \wedge y_{1}\right) \\
& \times p_{1}^{a_{1}}\left(s_{2}, y_{1} \mid s_{1}\right) \delta_{1}\left(a_{1} \mid s_{1}, \lambda\right)
\end{aligned}
$$

for $\left(s_{1}, \lambda\right) \in S_{1}$ and $\pi=\left(\delta_{1}, \delta_{2}, \delta_{3}\right) \in C_{1}^{3}$. We define optimal value functions $F_{n}^{*}$ for the imbedded subproblem by

$$
F_{n}^{*}(s, \lambda)=\sup _{\pi \in C_{n}^{N}} F_{n}^{\pi}(s, \lambda), \quad n \in \mathbf{N}
$$

for each $(s, \lambda) \in S_{1}$. Then we notice that optimal value in the original problem is

$$
F_{1}^{*}(s, M)=\sup _{\pi \in C_{1}^{N}} F_{1}^{\pi}(s, M)=\sup _{\pi \in C} E_{s}^{\pi}[Z],
$$

since $Z \leq M$. A policy $\pi$ is said to be optimal if $F_{1}^{*}(s, M)=F_{1}^{\pi}(s, M)$ for every $s \in S$.

We define the following sets of functions: let $\mathcal{F}$ be the set of functions $F$ from $S_{1}$ into an interval $[0, M]$ such that $F(s, 0)=0$ for each $s \in S$ and $F(s, \cdot)$ is measurable on $[0, M]$, and let $\mathcal{F}_{c}$ be the set of functions $F \in \mathcal{F}$ such that $F(s, \cdot)$ is nondecreasing and continuous on $[0, M]$ for each $s \in S$. In Theorem 3.1 it is shown that $F_{n}^{*} \in \mathcal{F}_{c}$.

We define operators $T_{n}^{a}, T_{n}^{\delta}$ and $T_{n}$ from $\mathcal{F}$ into itself as follows. For $F \in \mathcal{F}$, $(s, \lambda) \in S_{1}, a \in A(s), \delta \in \Delta_{M}$ and $1 \leq n \leq N-1$,

$$
\begin{aligned}
& T_{n}^{a} F(s, \lambda)=\sum_{s^{\prime} \in S} \sum_{y \in E} F\left(s^{\prime}, \lambda \wedge y\right) p_{n}^{a}\left(s^{\prime}, y \mid s\right), \\
& T_{n}^{\delta} F(s, \lambda)=\sum_{a \in A(s)} T_{n}^{a} F(s, \lambda) \delta(a \mid s, \lambda), \\
& T_{n} F(s, \lambda)=\sup _{\delta \in \Delta_{M}} T_{n}^{\delta} F(s, \lambda)=\max _{a \in A(s)} T_{n}^{a} F(s, \lambda)
\end{aligned}
$$

and

$$
T_{N}^{a} F(s, \lambda)=\sum_{y \in E} F(s, \lambda \wedge y) \tilde{p}_{N}^{a}(y \mid s),
$$




$$
\begin{aligned}
& T_{N}^{\delta} F(s, \lambda)=\sum_{a \in A(s)} T_{N}^{a} F(s, \lambda) \delta(a \mid s, \lambda), \\
& T_{N} F(s, \lambda)=\sup _{\delta \in \Delta_{M}} T_{N}^{\delta} F(s, \lambda)=\max _{a \in A(s)} T_{N}^{a} F(s, \lambda) .
\end{aligned}
$$

In all argument, for $F, G \in \mathcal{F}, F \geq G$ means that $F(s, \lambda) \geq G(s, \lambda)$ for all $(s, \lambda) \in$ $S_{1}$.

\section{Optimal value and optimal policy}

In this section we give an optimality equation by using operators $T_{n}$ and show that there exist a right continuous deterministic Markov policy.

We first give fundamental lemmas for operators $T_{n}^{a}, T_{n}^{\delta}$ and $T_{n}$.

Lemma 3.1. (i) For $F, G \in \mathcal{F}, \delta \in \Delta$ and $n \in \mathbf{N}, T_{n}^{\delta} F-T_{n}^{\delta} G=T_{n}^{\delta}(F-G)$.

(ii) Let $n \in \mathbf{N}$. If $F, G \in \mathcal{F}$ and $F \geq G$, then $T_{n}^{a} F \geq T_{n}^{a} G$ for each $a \in A(\cdot)$, $T_{n}^{\delta} F \geq T_{n}^{\delta} G$ for each $\delta \in \Delta$ and $T_{n} F \geq T_{n} G$.

(iii) Let $n \in \mathbf{N}$. If $F \in \mathcal{F}_{c}$, then $T_{n}^{a} F \in \mathcal{F}_{c}$ for any $a \in A(\cdot)$ and $T_{n} F \in \mathcal{F}_{c}$.

Proof. The statements (i) and (ii) are immediate results of definitions.

(iii) Let $F \in \mathcal{F}_{c}$ and let $s \in S$ be arbitrary. Then $F\left(s^{\prime}, 0\right)=0$ for every $s^{\prime} \in S$. Since $y \in[0, M]$, we have $T_{n}^{a} F(s, 0)=0$ for every $a \in A(s)$ and hence $T_{n} F(s, 0)=0$. It easily follows that $T_{n}^{a} F\left(s, \dot{)}\right.$ and $T_{n} F(s, \cdot)$ are nondecreasing on $[0, M]$, since $F\left(s^{\prime}, \cdot\right)$ is nondecreasing. Also, by the dominated convergence theorem we see that $T_{n}^{a} F(s, \cdot)$ is continuous on $[0, M]$ for each $a \in A(s)$, since $F\left(s^{\prime}, \cdot\right)$ is continuous. Thus since $A(s)$ is finite, $T_{n} F(s, \cdot)$ is also continuous on $[0, M]$. Therefore the proof is complete.

Lemma 3.2. Let $n \in \mathbf{N}$. For each $F \in \mathcal{F}_{c}$, there exists a right continuous decision rule $\delta \in \Delta_{D}$ satisfying $T_{n} F=T_{n}^{\delta} F$.

Proof. Let $F \in \mathcal{F}_{c}$ and $(s, \lambda) \in S_{1}$ be arbitrarily fixed. From Lemma 3.1 (iii), $T_{n}^{a} F(s, \cdot)$ is continuous on $[0, M]$ for each $a \in A(s)$. Since $A(s)$ is finite, we see that there exist $\mu>0$ and $a \in A(s)$ such that $T F(s, u)=T^{a} F(s, u)$ for all $u$ satisfying $\lambda \leq u<\lambda+\mu$. For such an action $a$, if we define $\delta \in \Delta_{D}$ by $\delta(s, u)=a$ for every $u$ so that $\lambda \leq u<\lambda+\mu$, then $\delta$ is right continuous and $T_{n} F(s, \lambda)=T_{n}^{\delta} F(s, \lambda)$. Therefore the proof is complete.

For any $\pi=\left(\delta_{n}, \delta_{n+1}, \ldots, \delta_{N}\right) \in C_{n}^{N}$ and a given history $(s, \lambda, a) \in S_{1} \times A$, the cut-head policy of $\pi$ to $(s, \lambda, a)$ is defined by ${ }^{1} \pi^{(s, \lambda, a)}=\left(\delta_{n+1}^{(s, \lambda, a)}, \delta_{n+2}^{(s, \lambda, a)}, \ldots, \delta_{N}^{(s, \lambda, a)}\right)$ where $\delta_{k+1}^{(s, \lambda, a)}\left(\cdot \mid h_{k}\right)=\delta_{k+1}\left(\cdot \mid(s, \lambda, a), h_{k}\right)$ for every $h_{k} \in H_{k-n+1}$ and each $k=n, n+$ $1, \ldots, N-1$. Then we see that ${ }^{1} \pi^{(s, \lambda, a)} \in C_{n+1}^{N}$ for a fixed $(s, \lambda, a)$. For the sake of simplicity we use a notation:

$$
T_{n}^{\delta_{n}} F_{n+1}^{1} \pi(s, \lambda)=\sum_{a \in A(s)} \delta_{n}(a \mid s, \lambda) \sum_{s^{\prime}, y} F_{n+1}^{1} \pi^{(s, \lambda, a)}\left(s^{\prime}, \lambda \wedge y\right) p_{n}^{a}\left(s^{\prime}, y \mid s\right)
$$

for $\pi=\left(\delta_{n}, \delta_{n+1}, \ldots, \delta_{N}\right) \in C_{n}^{N}$ and $(s, \lambda) \in S_{1}$.

Lemma 3.3. (i) For each $n \in \mathbf{N}$ and any $\pi \in C_{n}^{N}, F_{n}^{\pi} \in \mathcal{F}$.

(ii) Let $n=1,2, \ldots, N-1$ and let $\pi=\left(\delta_{n}, \delta_{n+1}, \ldots, \delta_{N}\right) \in C_{n}^{N}$ be arbitrary. Then $F_{n}^{\pi}=T_{n}^{\delta_{n}} F_{n+1}^{1 \pi}$. 
Proof. To show that $F_{n}^{\pi} \in \mathcal{F}$, it suffices to prove that $F_{n}^{\pi}(s, \cdot)$ is measurable on $[0,1]$ for each $s \in S$, since $F_{n}^{\pi}(s, 0)=E_{s}^{\pi}\left[0 \wedge Z_{n}\right]=0$ for every $s \in S$. For any $\pi=\left(\delta_{N}\right) \in C_{N}^{N}$ we have

$$
F_{N}^{\pi}(s, \lambda)=E_{s}^{\pi}\left[\lambda \wedge Z_{N}\right]=\sum_{a \in A(s)} \delta_{N}(a \mid s, \lambda) \sum_{y}(\lambda \wedge y) \tilde{p}_{N}^{a}(y \mid s) .
$$

Hence $F_{N}^{\pi}(s, \cdot)$ is measurable. We assume that $F_{n+1}^{1} \pi(s, \cdot)$ is measurable for $n \leq N-1$ and any ${ }^{1} \pi \in C_{n+1}^{N}$. Then for any $\pi=\left(\delta_{n}, \cdots, \delta_{N}\right) \in C_{n}^{N}$ we have

$$
T_{n}^{\delta_{n}} F_{n+1}^{1} \pi(s, \lambda)=\sum_{a \in A(s)} \delta_{n}(a \mid s, \lambda) \sum_{s^{\prime}, y} F_{n+1}^{1 \pi \pi(s, \lambda, a)}\left(s^{\prime}, \lambda \wedge y\right) p_{n}^{a}\left(s^{\prime}, y \mid s\right) .
$$

Thus $T_{n}^{\delta_{n}} F_{n+1}^{1} \pi(s, j$ is measurable. However it follows from Markov property that

$$
\begin{aligned}
& T_{n}^{\delta_{n}} F_{n+1}^{1} \pi(s, \lambda)=\sum_{a \in A(s)} \delta_{n}(a \mid s, \lambda) \sum_{s^{\prime}, y} E_{s^{\prime}}^{1} \pi(s, \lambda, a)\left[\lambda \wedge y \wedge Z_{n+1}\right] p_{n}^{a}\left(s^{\prime}, y \mid s\right) \\
& =E_{s}^{\pi}\left[\lambda \wedge Z_{n}\right] \\
& =F_{n}^{\pi}(s, \lambda) \text {. }
\end{aligned}
$$

Hence $F_{n}^{\pi}\left(s, \dot{)}\right.$ is measurable and we also have $T_{n}^{\delta_{n}} F_{n+1}^{1} \pi(s, \lambda)=F_{n}^{\pi}(s, \lambda)$. Therefore the proof is complete.

We next give a main theorem for optimal value functions and optimal policies in subproblems.

TheOREM 3.1. (i) For each $n \in \mathbf{N}, F_{n}^{*} \in \mathcal{F}_{c}$ and $\left\{F_{n}^{*}, n \in \mathbf{N}\right\}$ satisfies optimality equations:

$$
F_{n}^{*}=T_{n} F_{n+1}^{*}, 1 \leq n \leq N-1
$$

with $F_{N}^{*}(s, \lambda)=\max _{a \in A(s)} \sum_{y}(\lambda \wedge y) \tilde{p}_{N}^{a}(y \mid s)$.

(ii) For each $n \in \mathbf{N}$, there exists a right continuous policy $\pi \in C_{n}^{N} \cap C_{D}$ such that $F_{n}^{*}=F_{n}^{\pi}$.

Proof. We prove the statements (i) and (ii) by backward induction. When $n=N$, we see that

$$
\begin{aligned}
F_{N}^{*}(s, \lambda) & =\sup _{\pi \in C_{N}^{N}} E_{s}^{\pi}\left[\lambda \wedge Z_{N}\right] \\
& =\sup _{\delta_{N}} \sum_{a \in A(s)} \delta_{N}(a \mid s, \lambda) \sum_{y}(\lambda \wedge y) \tilde{p}_{N}^{a}(y \mid s) \\
& =\max _{a \in A(s)} \sum_{y}(\lambda \wedge y) \tilde{p}_{N}^{a}(y \mid s) .
\end{aligned}
$$

Since $\sum_{y}(\lambda \wedge y) \tilde{p}_{N}^{a}(y \mid s)$ is right continuous in $\lambda$, there is a right continuous decision rule $\delta_{N} \in \Delta_{D}$ such that

$$
F_{N}^{*}(s, \lambda)=\sum_{y}(\lambda \wedge y) \tilde{p}_{N}^{\delta_{N}(s, \lambda)}(y \mid s)=F_{N}^{\pi^{*}}(s, \lambda)
$$


where $\pi^{*}=\left(\delta_{N}\right) \in C_{N}^{N} \cap C_{D}$, and hence $F_{N}^{*} \in \mathcal{F}_{c}$. We assume that $F_{k}^{*} \in \mathcal{F}_{c}$ and there exist a right continuous policy $\sigma \in C_{k}^{N} \cap C_{D}$ such that $F_{k}^{*}=F_{k}^{\sigma}$. Then it follows from Lemma 3.2 that there is a right continuous decision rule $\hat{\delta}_{k-1} \in \Delta_{D}$ such that $T_{k-1} F_{k}^{*}=T_{k-1}^{\hat{\delta}_{k-1}} F_{k}^{*}$, which implies that $\pi=\left(\hat{\delta}_{k-1}, \sigma\right) \in C_{k-1}^{N} \cap C_{D}$ is a right continuous policy. It also follows from Lemma 3.3(ii) that

$$
F_{k-1}^{*}(s, \lambda) \geq F_{k-1}^{\pi}(s, \lambda)=T_{k-1}^{\hat{\delta}_{k-1}} F_{k}^{\sigma}(s, \lambda)=T_{k-1}^{\hat{\delta}_{k-1}} F_{k}^{*}(s, \lambda)=T_{k-1} F_{k}^{*}(s, \lambda)
$$

for each $(s, \lambda) \in S_{1}$. Conversely, we see from Lemma 3.3(ii) again that for any policy $\tau=\left(\delta_{k-1}, \delta_{k}, \ldots, \delta_{N}\right) \in C_{k-1}^{N}$,

$$
F_{k+1}^{\tau}(s, \lambda)=T_{k-1}^{\delta_{k-1}} F_{k}^{1} \tau(s, \lambda) \leq T_{k-1}^{\delta_{k-1}} F_{k}^{*}(s, \lambda) \leq T_{k-1} F_{k}^{*}(s, \lambda),
$$

since ${ }^{1} \tau \in C_{k}^{N}$. Taking supremum over $C_{k-1}^{N}$, we obtain $F_{k-1}^{*}(s, \lambda) \leq T_{k-1} F_{k}^{*}(s, \lambda)$. Thus, combining with the previous inequality, we have $T_{k-1} F_{k}^{*}=F_{k-1}^{*}=F_{k-1}^{\pi}$. Hence, $\pi$ satisfies $F_{k-1}^{*}=F_{k-1}^{\pi}$, and from Lemma 3.1(iii), we have $F_{k-1}^{*} \in \mathcal{F}_{c}$. By backward induction, the proof of the statements (i) and (ii) is complete.

We finally find optimal function and optimal policy in the original problem.

ThEOREM 3.2. Optimal value $F_{1}^{*}(s, M)$ is given by

$$
F_{1}^{*}=T_{1} T_{2} \cdots T_{N-1} F_{N}^{*}
$$

with $F_{N}^{*}(s, r)=\max _{a \in A(s)} \sum_{y}(\lambda \wedge y) \tilde{p}_{N}^{a}(y \mid s)$, and a right continuous optimal policy $\pi=\left(\delta_{1}, \delta_{2}, \ldots, \delta_{n}\right) \in C_{D}$ is obtained by

$$
\begin{aligned}
& T_{n} F_{n+1}^{*}=T_{n}^{\delta_{n}} F_{n+1}^{*}, 1 \leq n \leq N-1, \\
& F_{N}^{*}(s, \lambda)=\sum_{y}(\lambda \wedge y) \tilde{p}_{N}^{\delta_{N}(s, \lambda)}(y \mid s)=\max _{a \in A(s)} \sum_{y}(\lambda \wedge y) \tilde{p}_{N}^{a}(y \mid s),(s, \lambda) \in S_{1} .
\end{aligned}
$$

PROOF. These are immediate results of Theorem 3.1 and its proof.

\section{A numerical example}

We give a simple numerical example of two stages, two states and two actions in this section.

Let state space be $S=\left\{s_{1}, s_{2}\right\}$ and let action space be $A\left(s_{1}\right)=A\left(s_{2}\right)=\left\{a_{1}, a_{2}\right\}$ and let $N=2$. We assume that probability distributions $p_{1}^{a}\left(s^{\prime}, y \mid s\right)$ and $\tilde{p}_{2}^{a}(y \mid s)$ are determined by

$$
\begin{aligned}
& p_{1}^{a_{1}}\left(s_{1}, y \mid s_{1}\right)=\left\{\begin{array}{cc}
\frac{1}{4} & \text { if } y=1 \\
\frac{1}{4} & \text { if } y=3
\end{array}, \quad p_{1}^{a_{1}}\left(s_{2}, 2 \mid s_{1}\right)=\frac{1}{2},\right. \\
& p_{1}^{a_{2}}\left(s_{1}, \frac{8}{5} \mid s_{1}\right)=\frac{2}{5}, \quad p_{1}^{a_{2}}\left(s_{2}, y \mid s_{1}\right)=\left\{\begin{array}{ll}
\frac{1}{5} & \text { if } y=\frac{6}{5} \\
\frac{2}{5} & \text { if } y=\frac{3}{2}
\end{array}\right. \text {, } \\
& p_{1}^{a_{1}}\left(s_{1}, \frac{5}{4} \mid s_{2}\right)=1, \quad p_{1}^{a_{2}}\left(s_{2}, 3 \mid s_{2}\right)=1, \\
& \tilde{p}_{2}^{a_{1}}\left(y \mid s_{1}\right)=\left\{\begin{array}{ll}
\frac{2}{5} & \text { if } y=3 \\
\frac{3}{5} & \text { if } y=2
\end{array}, \quad \tilde{p}_{2}^{a_{1}}\left(y \mid s_{2}\right)=\left\{\begin{array}{ll}
\frac{1}{2} & \text { if } y=1 \\
\frac{1}{2} & \text { if } y=2
\end{array},\right.\right. \\
& \tilde{p}_{2}^{a_{2}}\left(y \mid s_{1}\right)=\left\{\begin{array}{cl}
\frac{3}{10} & \text { if } y=\frac{3}{2} \\
\frac{7}{10} & \text { if } y=3
\end{array}, \quad \tilde{p}_{2}^{a_{2}}\left(y \mid s_{2}\right)=\left\{\begin{array}{ll}
\frac{4}{5} & \text { if } y=1 \\
\frac{1}{5} & \text { if } y=4
\end{array} .\right.\right.
\end{aligned}
$$


Then we shall first give optimal value $F_{2}^{*}$ and optimal decision $\delta_{2}$ at $N=2$. Since $F_{2}^{*}(s, \lambda)=\max _{a \in A(s)} \sum_{y}(\lambda \wedge y) \tilde{p}_{2}^{a}(y \mid s)$ by Theorem 3.1, we have

$$
\begin{aligned}
F_{2}^{*}\left(s_{1}, \lambda\right)= & \max \left(\frac{2}{5}(\lambda \wedge 3)+\frac{3}{5}(\lambda \wedge 2), \frac{3}{10}\left(\lambda \wedge \frac{3}{2}\right)+\frac{7}{10}(\lambda \wedge 3)\right) \\
= & \begin{array}{ll}
\lambda & \text { if } \lambda<2 \\
\frac{2}{5} \lambda+\frac{6}{5} & \text { if } 2 \leq \lambda<\frac{5}{2} \\
\frac{7}{10} \lambda+\frac{9}{20} & \text { if } \frac{5}{2} \leq \lambda<3 \\
\frac{51}{20} & \text { if } \lambda \geq 3
\end{array}
\end{aligned}
$$

and

$$
\begin{aligned}
F_{2}^{*}\left(s_{2}, \lambda\right)= & \max \left(\frac{1}{2}(\lambda \wedge 1)+\frac{1}{2}(\lambda \wedge 2), \frac{4}{5}(\lambda \wedge 1)+\frac{1}{5}(\lambda \wedge 4)\right) \\
= & \begin{array}{ll}
\lambda & \text { if } \lambda<1 \\
\frac{1}{2} \lambda+\frac{1}{2} & \text { if } 1 \leq \lambda<2 \\
\frac{3}{2} & \text { if } 2 \leq \lambda<\frac{7}{2} \\
\frac{1}{5} \lambda+\frac{4}{5} & \text { if } \frac{7}{2} \leq \lambda<4 \\
\frac{8}{5} & \text { if } \lambda \geq 4
\end{array}
\end{aligned}
$$

Hence we have

$$
\delta_{2}\left(s_{1}, \lambda\right)=\left\{\begin{array}{ll}
a_{1} \text { or } a_{2} & \text { if } \lambda<\frac{3}{2} \\
a_{1} & \text { if } \frac{3}{2} \leq \lambda<\frac{5}{2} \\
a_{2} & \text { if } \lambda \geq \frac{5}{2}
\end{array}, \quad \delta_{2}\left(s_{2}, \lambda\right)=\left\{\begin{array}{ll}
a_{1} \text { or } a_{2} & \text { if } \lambda<1 \\
a_{1} & \text { if } 1 \leq \lambda<\frac{7}{2} \\
a_{2} & \text { if } \lambda \geq \frac{7}{2}
\end{array} .\right.\right.
$$

Next we consider optimal $F_{1}^{*}$ and $\delta_{1}$ at the first stage. From Theorem 3.1, we have

$$
\begin{aligned}
F_{1}^{*}(s, \lambda) & =T_{1} F_{2}^{*}(s, \lambda)=\max _{a \in A(s)} T_{1}^{a} F_{2}^{*}(s, \lambda) \\
& =\max _{a \in A(s)} \sum_{s^{\prime} \in S} \sum_{y \in E} F_{2}^{*}\left(s^{\prime}, \lambda \wedge y\right) p_{1}^{a}\left(s^{\prime}, y \mid s\right) .
\end{aligned}
$$

By the way, we easily see that

$$
\begin{aligned}
T_{1}^{a_{1}} F_{2}^{*}\left(s_{1}, \lambda\right)= & \frac{1}{4} F_{2}^{*}\left(s_{1}, \lambda \wedge 1\right)+\frac{1}{4} F_{2}^{*}\left(s_{1}, \lambda \wedge 3\right)+\frac{1}{2} F_{2}^{*}\left(s_{2}, \lambda \wedge 2\right) \\
= & \begin{cases}\lambda & \text { if } \lambda<1 \\
\frac{1}{2} \lambda+\frac{1}{2} & \text { if } 1 \leq \lambda<2 \\
\frac{1}{10} \lambda+\frac{13}{10} & \text { if } 2 \leq \lambda<\frac{5}{2} \\
\frac{7}{40} \lambda+\frac{89}{80} & \text { if } \frac{5}{2} \leq \lambda<3 \\
\frac{131}{80} & \text { if } \lambda \geq 3\end{cases}
\end{aligned}
$$


and

$$
\begin{aligned}
& T_{1}^{a_{2}} F_{2}^{*}\left(s_{1}, \lambda\right)=\frac{2}{5} F_{2}^{*}\left(s_{1}, \lambda \wedge \frac{8}{5}\right)+\frac{2}{5} F_{2}^{*}\left(s_{2}, \lambda \wedge \frac{3}{2}\right)+\frac{1}{5} F_{2}^{*}\left(s_{2}, \lambda \wedge \frac{6}{5}\right) \\
& =\left\{\begin{array}{ll}
\lambda & \text { if } \lambda<1 \\
\frac{7}{10} \lambda+\frac{3}{10} & \text { if } 1 \leq \lambda<\frac{6}{5} \\
\frac{3}{5} \lambda+\frac{21}{50} & \text { if } \frac{6}{5} \leq \lambda<\frac{3}{2} \\
\frac{2}{5} \lambda+\frac{18}{25} & \text { if } \frac{3}{2} \leq \lambda<\frac{8}{5} \\
\frac{34}{25} & \text { if } \lambda \geq \frac{8}{5}
\end{array} .\right.
\end{aligned}
$$

Hence we have optimal value in the imbedded problem as follows :

$$
\begin{aligned}
F_{1}^{*}\left(s_{1}, \lambda\right)= & \max _{a} T_{1}^{a} F_{2}^{*}\left(s_{1}, \lambda\right) \\
= & \begin{cases}T_{1}^{a_{1}} F_{2}^{*}\left(s_{1}, \lambda\right)=T_{1}^{a_{2}} F_{2}^{*}\left(s_{1}, \lambda\right) & \text { if } \lambda<1 \\
T_{1}^{a_{2}} F_{2}^{*}\left(s_{1}, \lambda\right) & \text { if } 1 \leq \lambda<\frac{43}{25} \\
T_{1}^{a_{1}} F_{2}^{*}\left(s_{1}, \lambda\right) & \text { if } \lambda \geq \frac{43}{25}\end{cases} \\
= & \begin{array}{ll}
\lambda & \text { if } \lambda<1 \\
\frac{7}{10} \lambda+\frac{3}{10} & \text { if } 1 \leq \lambda<\frac{6}{5} \\
\frac{3}{5} \lambda+\frac{21}{50} & \text { if } \frac{6}{5} \leq \lambda<\frac{3}{2} \\
\frac{2}{5} \lambda+\frac{18}{25} & \text { if } \frac{3}{2} \leq \lambda<\frac{8}{5} \\
\frac{34}{25} & \text { if } \frac{8}{5} \leq \lambda<\frac{43}{25} \\
\frac{1}{2} \lambda+\frac{1}{2} & \text { if } \frac{43}{25} \leq \lambda<2 \\
\frac{1}{10} \lambda+\frac{13}{10} & \text { if } 2 \leq \lambda<\frac{5}{2} \\
\frac{7}{40} \lambda+\frac{89}{80} & \text { if } \frac{5}{2} \leq \lambda<3 \\
\frac{131}{80} & \text { if } \lambda \geq 3
\end{array}
\end{aligned}
$$

and optimal decision at state $s_{1}$ is

$$
\delta_{1}\left(s_{1}, \lambda\right)=\left\{\begin{array}{ll}
a_{1} \text { or } a_{2} & \text { if } \lambda<1 \\
a_{2} & \text { if } 1 \leq \lambda<\frac{43}{25} \\
a_{1} & \text { if } \lambda \geq \frac{43}{25}
\end{array} .\right.
$$

Similarly, optimal value at state $s_{2}$ is

$$
F_{1}^{*}\left(s_{2}, \lambda\right)=\left\{\begin{array}{ll}
\lambda & \text { if } \lambda<\frac{5}{4} \\
\frac{5}{4} & \text { if } \frac{5}{4} \leq \lambda<\frac{3}{2} \\
\frac{1}{2} \lambda+\frac{1}{2} & \text { if } \frac{3}{2} \leq \lambda<2 \\
\frac{3}{2} & \text { if } \lambda \geq 2
\end{array}\right. \text {, }
$$


since we easily have

$$
T_{1}^{a_{1}} F_{2}^{*}\left(s_{2}, \lambda\right)=\left\{\begin{array}{ll}
\lambda & \text { if } \lambda<\frac{5}{4} \\
\frac{5}{4} & \text { if } \lambda \geq \frac{5}{4}
\end{array}, \quad T_{1}^{a_{2}} F_{2}^{*}\left(s_{2}, \lambda\right)= \begin{cases}\lambda & \text { if } \lambda<1 \\
\frac{1}{2} \lambda+\frac{1}{2} & \text { if } 1 \leq \lambda<2 \\
\frac{3}{2} & \text { if } \lambda \geq 2\end{cases}\right.
$$

and optimal decision is

$$
\delta_{1}\left(s_{2}, \lambda\right)=\left\{\begin{array}{ll}
a_{1} \text { or } a_{2} & \text { if } \lambda<1 \\
a_{1} & \text { if } 1 \leq \lambda<\frac{3}{2} \\
a_{2} & \text { if } \lambda \geq \frac{3}{2}
\end{array} .\right.
$$

Therefore, since $M=4$, we have optimal value in the original problem as follows:

$$
F_{1}^{*}\left(s_{1}, M\right)=\frac{131}{80}, \quad F_{1}^{*}\left(s_{2}, M\right)=\frac{3}{2}
$$

\section{Acknowledgement}

The author is grateful to the referee for valuable remarks and helpful comments.

\section{References}

Bellman, R.E. and Zadeh, L.A (1970). Decision-making in a fuzzy environment, Management Science. 17, B141-B164.

Iwamoto, S. and Fujita, T. (1995). Stochastic decision-making in a fuzzy environment, J. Operations Research Society of Japan. 38, 467-482.

Iwamoto, S., Tsurusaki, K. and Fujita, T. (1999). Conditional decision-making in fuzzy environment, J. Operations Research Society of Japan. 42, 198-218.

Iwamoto, S., Tsurusaki, K. and Fujita, T. (2001). On Markov policies for minimax decision processes, J. Math. Anal. Appl. 253, 58-78.

Kacprzyk, J. (1978). Decision-making in a fuzzy environment with fuzzy termination time, Fuzzy Sets and Systems. 1, 169-179.

Esogbue, A.O. and Bellman, R.E. (1984). Fuzzy dynamic programming and its extensions, TIMS/Studies in Management Sciences. 20, 147-167.

White, D.J. (1993a). Minimizing a threshold probability in discounted Markov decision processes, J. Math. Anal. Appl. 173, 634-646.

Wu, C. and Lin, Y. (1999). Minimizing risk models in Markov decision processes with policies depending on target values, J. Math. Anal. Appl. 231, 47-67.

Ohtsubo, Y. and Toyonaga, K. (2002). Optimal policy for minimizing risk models in Markov decision processes, J. Math. Anal. Appl. 271, 66-81. 
Ohtsubo, Y. (2003). Minimizing risk models in stochastic shortest path problems, Math. Meth. Oper. Res. 57, 79-88.

Howard, R.A. (1960). Dynamic Programming and Markov Processes, Wiley, London.

White, D.J. (1993b). Markov Decision Processes, Wiley, New York.

Received August 26, 2004

Revised April 27, 2005 